(An ISO 3297: 2007 Certified Organization)

Vol. 3, Issue 8, August 2014

\title{
Wireless Sensor Network for Electric Meter Handling
}

\author{
Swapnil Magar ${ }^{1}$, Prof.Deshpande L.M. ${ }^{2}$ \\ PG Student [E\&TC], Dept. of ECE, College of Engineering, Osmanabad, Maharashtra, India ${ }^{1}$ \\ Assistant professor, Dept. of ECE, College of Engineering, Osmanabad, Maharashtra, India ${ }^{2}$
}

\begin{abstract}
The technology called wireless sensor network is the most efficient technology which can be used in various applications because of its low power consumption, simple to use, cheaper communication network. In this paper we present a model which is used for collecting and make billing of our monthly used electricity by using wireless sensor network. WSN is a network used to collection of widely distributed sensors which monitors energy used at each home or meter and cooperatively passes their data through the network to a centre location. It is a current most interesting topic of automation system. This system consists of meters, relay, a ZigBee operated wireless sensor network and GSM. GSM module used in this system is used to advance its characteristics such as sending bills, sending notices, due dates of payments to users. This system performs tasks such as taking meter reading, distribution of bills, sending notice, disconnection and reconnection of energy automatically. This model can lead to great deal of costs saving in electricity metering.
\end{abstract}

KEYWORDS: Micro-controller, GSM, Wireless Sensor Network, ZigBee.

\section{I.INTRODUCTION}

The traditional metering systems consist of manmade system, where meter readings are taken manually. But these systems has many limitations like, errors in reading, inaccuracy, delayed work, external conditions for affecting readings like reliability of the agents. These techniques also require huge manpower. And also for cutting the connection of energy for that user who is not paying electricity bills regularly.

One emerging technology called wireless sensors are capable of to overcome all above limitations. And also eliminate disadvantages in traditional networks in which, the sensor/actuator network is connected with physical world directly, then store data in data base and control action is taken by human interface. But by using WSN, this network senses physical information, sends it to control station, decides control action based on its observations automatically and performs suitable actions. The investments on manual meter reading are eliminated and the collected data becomes more accurate.

\section{A. Objective of This Study}

The objective of this study is to propose a new wireless technology alternative for automatic meter reading for the Provincial Electricity Authority. The technology chosen is ZigBee which is a new wireless network technology that has just been developed for remote metering application. This study shows characteristics of ZigBee technology, equipments requirement for AMR, a conceptual design for pilot project, and benefits from implementing this technology.

\section{B.Benefits of AMR system.}

Benefits gained from AMR system can be divided into three parts, which are benefits for electricity utility service providers, benefits for customers and benefits for the country.

\section{- For electricity utility service providers:}

- Reduce data collection costs 


\section{8 \\ International Journal of Advanced Research in Electrical, Electronics and Instrumentation Engineering \\ (An ISO 3297: 2007 Certified Organization)}

\section{Vol. 3, Issue 8, August 2014}

- Improve meter reading accuracy

- Enable faster, more efficient reading times and billing process

- Monitor overall energy consumption

- Significantly increase operational efficiency by providing real time pricing.

- Large number of meters can be managed from a single point of presence

- Improve customer service

- Enable conservation of resources

- Estimate usage on a daily or hourly basis and forecast total demand more precisely

- Reduce technical and commercial losses

- Real-time meter status monitoring

\section{- For customers:}

- Monitor energy consumption

- Enable demand-side energy management

- Enable energy saving and money saving

- Improved customer service

\section{- For the country:}

- Support nation's electricity energy efficiency scheme

- Help reducing electricity consumption and increasing natural resources utilization [1].

\section{II.SYSTEM MODEL AND ASSUMPTIONS}

\section{A.System Architecture}

This model is creates a electricity distribution network where each meter is connected to the central office through WSN. Each meter consists of a controller \& a communication module where the controller is used to measuring energy usage continuously and stores it in its internal memory. And communication module sends the total energy usage by meter periodically to the central office for further action.

At the central office incoming data is store in database at office on personal address allotted to each user. Then after every month from central computer SMS is send to the user personal contact number and emailed to personal mail id stored in his/her personal database automatically. Also the due date is also being sent with bill amount.

Also there is a facility added to this proposed model is that, if the customer is not paying the bill after due date we can able to disconnect connection to that particular user/customer. Also we can add a facility to customers that if they do not need energy for 10 to 15 days or more then the can request to disconnect energy for that period. Fig 1 . Shows architecture of system

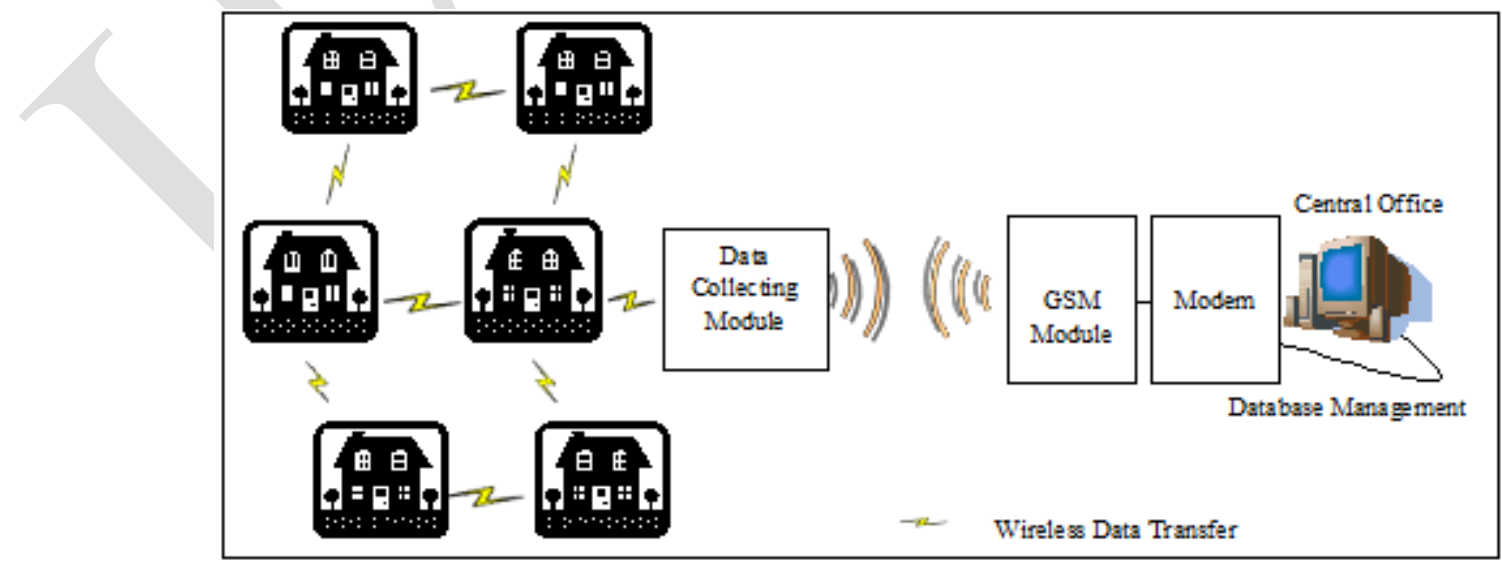

. Figure 1: Architecture of System 
 Electronics and Instrumentation Engineering \\ (An ISO 3297: 2007 Certified Organization) \\ Vol. 3, Issue 8, August 2014}

\section{B. System Block Diagram}

The figure 2 shows Block diagram of one sensor node, which consists of

- Sensing part

- Controlling part using microcontroller

- Transceivers part

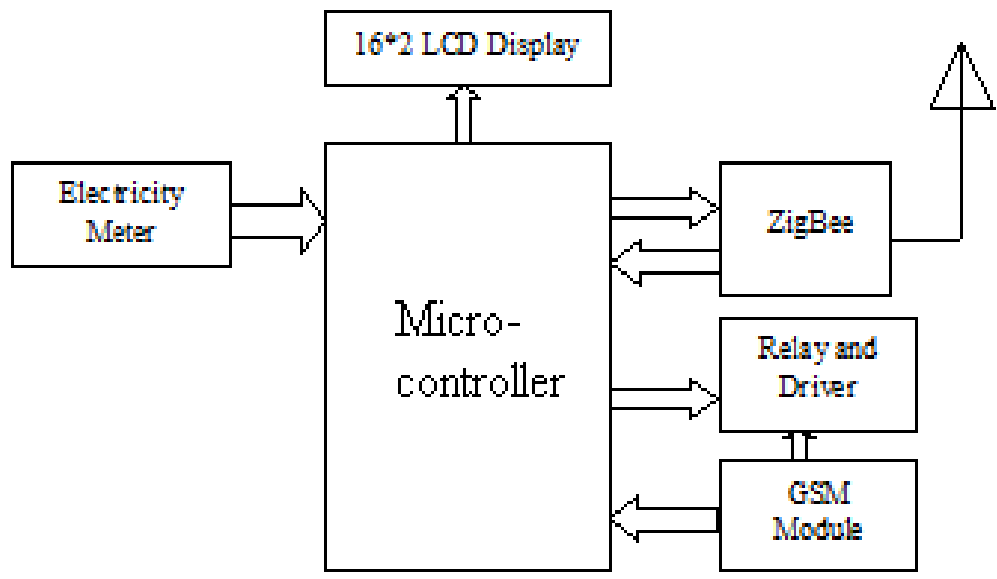

Figure 2: Block Diagram of System

\section{A. Sensing Part}

Basic unit of system is sensing part which consists of an electricity meter, which is used for take readings from individual houses. An electricity meter or energy meter is a device that measures the amount of electric energy consumed by a household, industrial, or an electrically powered device. Electricity meters are calibrated in billing units typically in the kilowatt hour $[\mathrm{kWh}]$ and operate by continuously measuring the instantaneous voltage (volts) and current (amperes) to give energy used (in joules, kilowatt-hours etc.). Single-Phase Power/Energy Meter with Tamper Detection is used for detecting amount energy consumed. This electricity meters give Energy Pulse Output in the form of led blink which goes beyond 32 pulses / $\mathrm{kWh}$.These pulses are given directly to microcontroller.

\section{B. Controlling Part}

Controlling part consists of Microcontroller which performs following functions:

- collection, managing and storage of data from the sensors

- Interfacing the sensor data to the communication medium.

The microcontroller is connected to LCD for continuous display of value of electricity consumed. Controlling part is also included in the module by connecting the microcontroller through the relay driver and relay which cuts and resumes supply if bill payment is not done.

The AT89C51 is a low-power, high-performance CMOS 8-bit microcomputer with 4K

Bytes of Flash programmable and erasable read only memory (PEROM). The device is manufactured using Atmel's high-density non-volatile memory technology and is compatible with the industry-standard MCS-51 instruction set and pin out. The on-chip Flash allows the program memory to be reprogrammed in-system or by a conventional nonvolatile memory programmer. By combining a versatile 8-bit CPU with Flash on a monolithic chip, the Atmel AT89C51 is a powerful microcomputer which provides a highly-flexible and cost-effective solution to many embedded control applications.[2] 


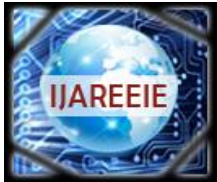 \\ ISSN (Print) : $2320-3765$ \\ ISSN (Online): 2278 - 8875 \\ International Journal of Advanced Research in Electrical, Electronics and Instrumentation Engineering
}

\author{
(An ISO 3297: 2007 Certified Organization)
}

\section{Vol. 3, Issue 8, August 2014}

The AT89C51 provides the following standard features: 4K bytes of Flash, 128 bytes of RAM, 32 I/O lines, two 16-bit timer/counters, five vector two-level interrupt architecture, a full duplex serial port, on-chip oscillator and clock circuitry. In addition, the AT89C51 is designed with static logic for operation down to zero frequency and supports two software selectable power saving modes. The Idle Mode stops the CPU while allowing the RAM, timer/counters, serial port and interrupt system to continue functioning. The Power-down Mode saves the RAM contents but freezes the oscillator disabling all other chip functions until the next hardware reset.[2]

\section{III.EFFICIENT COMMUNICATION}

\section{A. Transceivers: ZigBee Technology}

ZigBee is the name of a specification for a suite of high level communication protocols using small, lowpower digital radios based on the IEEE 802.15.4 standard for wireless personal area networks (WPANs) first announced in year 2004. ZigBee is targeted at radio frequency (RF) applications that require a low data rate, low-power consumption, and secure networking. ZigBee operates in the industrial, scientific and medical (ISM) radio bands; 868 $\mathrm{MHz}$ in Europe, $915 \mathrm{MHz}$ in the USA and $2.4 \mathrm{GHz}$ in most jurisdictions worldwide, including Thailand. The technology is intended to be simpler and cheaper than other WPANs such as Bluetooth[1].

ZigBee protocols are intended for use in embedded applications requiring low data rates and low power consumption. ZigBee's current focus is to define a general purpose, inexpensive, self-organizing, mesh network that can be used for industrial control, embedded sensing, medical data collection, smoke and intruder warning, building automation, home automation, etc. The resulting network will use very small amounts of power so individual devices might run for a year or two using the originally installed battery [1].

Even though the ZigBee data rate is only $250 \mathrm{kbps}$, it is adequate for AMR as the data acquired each time from a meter has a size of only 1,000 Bytes (8,000 bits)[1].

\section{B. GSM Module}

The development of GSM started in early 1980's for Europe's Mobile infrastructure. The first was to establish a team with the title "Group Special Mobile" (hence the term "GSM", which today stands for Global System for Mobile Communications) to develop a set of common standards. 1986 - A decision was reached to implement a digital transmission system.

The GSM standard was developed to replace first generation (1G) analog cellular networks. The GSM is an architecture used in most of the countries for mobile communication for exchanging and transmitting data in groups. It shares wireless channel in high speed and in distance.

GSM module is used to establish communication between a household energy meter/customer and the computer present at central office. GSM module consists of a GSM modem assembled together with power supply circuit and communication interfaces (like RS-232, USB, etc) for easy connection to computer or microcontroller based system. The power supply circuit is also built in the module. In this model GSM is used to communicate between user and central station. Data is transferred from meter in each and every house to data collecting module through ZigBee and then it is transferred to central station via GSM. And also the same GSM is used to communicate to every user to send the monthly bill amount \& due date to customer and receive any request from customer.

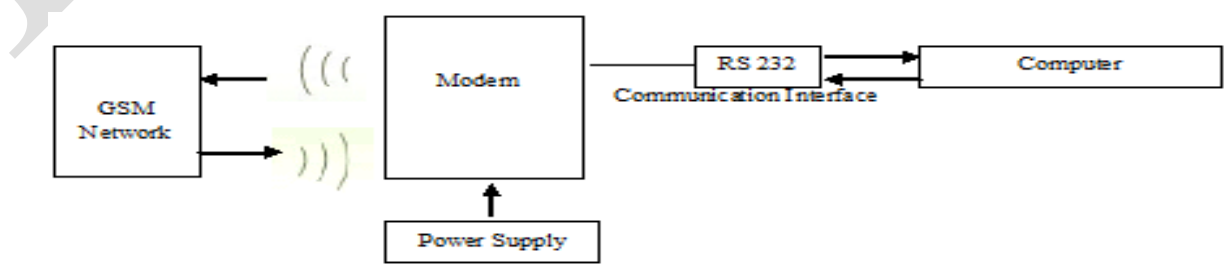


 Electronics and Instrumentation Engineering \\ (An ISO 3297: 2007 Certified Organization) \\ Vol. 3, Issue 8, August 2014 \\ V. RESULT AND DISCUSSION}

In this section, we discuss the results of the proposed system. At the meter end/house the microprocessor calculates energy usage by user in the form of $\mathrm{kWh}$ and store in the internal memory as well as sends to central office via communication system network (ZigBee \& GSM). Similarly the used energy units are display on the LCD continuously.

The data regarding energy consumption is send to central computer via GSM module for further processing. The central computer get that data \& store it in the central database where each user personal database is created which consists of name, address, mobile number, email etc.

After completion of month the central computer create a bill for every meter in three different manner as print out of bill, sends a SMS and sends a email to each user as shown in figs below.
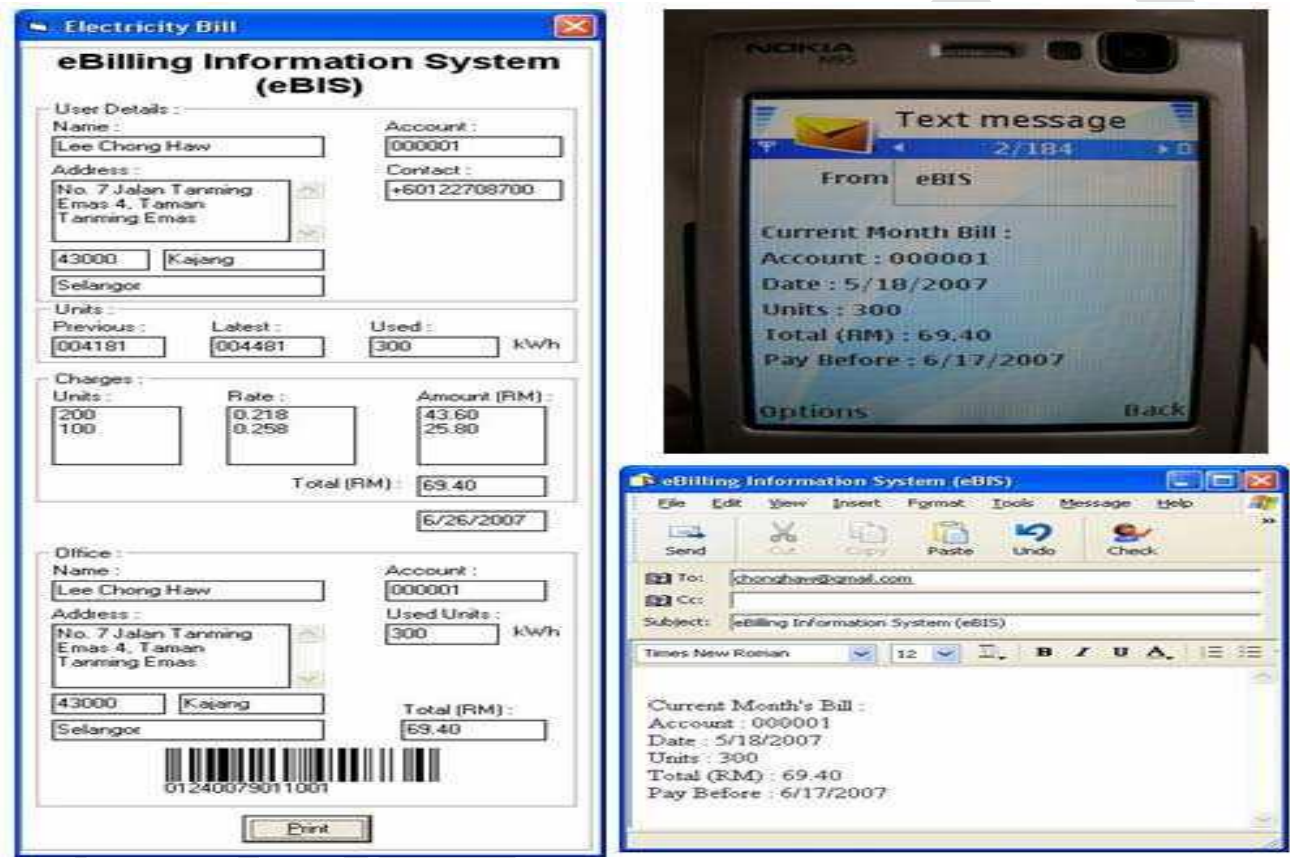

\section{VI.ADVANTAGES AND FUTURE SCOPE}

The system designed reduces the efforts of manual data collection of energy meter. Also, data which is received at service provider side is easy to manipulate for bill generation and other such tasks. With this system we can collect the reading as well as control the supply. With addition of software at service provider side, the customer can be informed of current meter reading \& bill, status of the line and other parameters to the customer with either message or a phone call.

\section{CONCLUSION}

With this system the service provider can collect the bill any time with a single message. The data collection and manipulation task becomes fast and easier. Any modification can be made to the code in less time. Changes in rate or unit calculation can be done very effectively. 


\section{(9) \\ ISSN (Print) : $2320-3765$ \\ ISSN (Online): $2278-8875$ \\ International Journal of Advanced Research in Electrical, Electronics and Instrumentation Engineering \\ (An ISO 3297: 2007 Certified Organization) \\ Vol. 3, Issue 8, August 2014 \\ REFERENCES}

1. “Feasibility study of zigbee-based automatic meter reading system for the provincial electricity authority”,By Joon Joongwong, Shinawatra University.

2. Babak Aghaei.B, Using Wireless Sensor Network in Water, Electricity and Gas industry, 978-1-4244-8679-3/11/\$26.00 @2011 IEEE

3. Hybrid System Automatic Meter Reading, by Dr. Mohd Yunus B Nayan, Aryo Handoko Primicanta2

4. Wireless Automatic Water-meter Reading System, byGordan Štruklec, Vedran Bilas

5. Design of remote automatic meter reading system based on ZigBee and GPRS,by Li Quan-Xi1, Li Gang2, ISBN 978-952-5726-10-Proceedings of the Third International Symposium on Computer Science and Computational Technology(ISCSCT '10)Jiaozuo, P. R. China, 14-15,August 2010, pp. 186-189

6. IEEE Std 802.15.14: Wireless Medium Access Control (MAC) and Physical Layer (PHY) Specifications for Low- Rate Wireless Personal Area Networks (LR-WPANs), 2003

7. LPC2131/32/34/36/38 Single-chip 16/32-bit microcontrollers; 32/64/128/256/512 kB ISP/IAP flash with 10-bit ADC and DAC Rev. 03 — 21 September 2006 Product data sheet

8. A. Iwayemi, and C. Zhou, "Developing ZigBee deployment guideline under WiFi interference for smart grid applications,"IEEE Transactions on Smart Grid, vol. 2, Page no.98-108, 2011

9. H. Farhangi, "The path of the smart grid," IEEE Power and Energy Magazine, vol. 8, pp. 18-28, 2010.

10. J. A. Gutierrez, "On the use of IEEE Std. 802.15.4 to enable wireless sensor networks in building automation,"

11. "Automatic Meter Reading System Based on Wireless Mesh Networks and SOPC Technology," Liting Cao; Wei Jiang; Zhaoli Zhang; , Intelligent Networks and Intelligent Systems, 2009. ICINIS '09. Second International Conference on, vol., no., pp.142-145, 1-3 Nov. 2009 doi: 10.1109/ICINIS.2009.45

12. www.atmel.in 